ARTICLE

\title{
ZNF143 mediates CTCF-bound promoter-enhancer loops required for murine hematopoietic stem and progenitor cell function
}

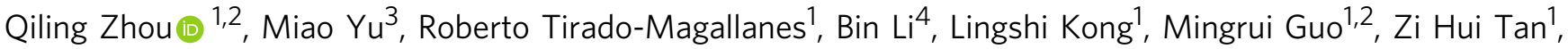
Sanghoon Lee ${ }^{1,12}$, Li Chai ${ }^{5}$, Akihiko Numata1,6, Touati Benoukraf (1) 1,7, Melissa Jane Fullwood 1,8,9,

Motomi Osato (10) 1 , Bing Ren (10) ${ }^{4,10}$ \& Daniel G. Tenen (10 1,11凶

CCCTC binding factor (CTCF) is an important factor in the maintenance of chromatin-chromatin interactions, yet the mechanism regulating its binding to chromatin is unknown. We demonstrate that zinc finger protein 143 (ZNF143) is a key regulator for CTCFbound promoter-enhancer loops. In the murine genome, a large percentage of CTCF and ZNF143 DNA binding motifs are distributed $37 \mathrm{bp}$ apart in the convergent orientation. Furthermore, deletion of ZNF143 leads to loss of CTCF binding on promoter and enhancer regions associated with gene expression changes. CTCF-bound promoter-enhancer loops are also disrupted after excision of ZNF143. ZNF143-CTCF-bound promoter-enhancer loops regulate gene expression patterns essential for maintenance of murine hematopoietic stem and progenitor cell integrity. Our data suggest a common feature of gene regulation is that ZNF143 is a critical factor for CTCF-bound promoter-enhancer loops.

\footnotetext{
${ }^{1}$ Cancer Science Institute of Singapore, National University of Singapore, 117599 Singapore, Singapore. ${ }^{2}$ YLL School of Medicine, National University of Singapore, 119228 Singapore, Singapore. ${ }^{3}$ School of Life Sciences, Fudan University, Shanghai, China. ${ }^{4}$ Ludwig Institute for Cancer Research, La Jolla, CA 92093, USA. ${ }^{5}$ Department of Pathology, Brigham and Women's Hospital, Boston, MA 02115, USA. ${ }^{6}$ Medicine and Biosystemic Science, Kyushu University Graduate School of Medical Science, Fukuoka, Japan. ${ }^{7}$ Division of BioMedical Sciences, Faculty of Medicine, Memorial University of Newfoundland, St. John's, NL A1B 3V6, Canada. ${ }^{8}$ School of Biological Sciences, Nanyang Technological University, 60 Nanyang Drive, 637551 Singapore, Singapore. ${ }^{9}$ Institute of Molecular and Cell Biology, Agency for Science, Technology and Research (A*STAR), 61 Biopolis Drive, Proteos 138673, Singapore. ${ }^{10}$ Department of Cellular \& Molecular Medicine, Moores Cancer Center and Institute of Genome Medicine, UCSD School of Medicine, 9500 Gilman Drive, La Jolla, CA 92093, USA. ${ }^{11}$ Harvard Stem Cell Institute, Harvard Medical School, Boston, MA 02115, USA. ${ }^{12}$ Present address: Department of Systems Biology, The University of Texas MD Anderson Cancer Center, Houston, TX 77030, USA. ${ }_{\text {email: daniel.tenen@nus.edu.sg }}$
} 
G ene transcription is commonly regulated by chromatin loops between promoters and regulatory elements such as enhancers and silencers ${ }^{1-3}$. Different transcription factors (TFs) bind to these regulatory elements to regulate promoter-enhancer loops in a cell-type-specific manner. Although they play an essential role in control of gene expression, how TFs contribute to promoter-enhancer loops is not well understood.

In addition to its essential role in maintaining Topologically Associating Domains (TADs) ${ }^{4-9}$, several lines of evidence suggest that CCCTC binding factor (CTCF) plays a critical role in promoter-enhancer loop regulation as well. First, downregulation of CTCF by either targeted degradation or RNAi decreases the intensity of enhancer-promoter loops, consistent with high enrichment of CTCF motifs within promoter regions ${ }^{4,5,7-10}$. Second, detection of CTCF in protein complexes enriched on active promoters by ChIP-MS supports that CTCF is involved in enhancer-promoter loops ${ }^{11}$.

While it has been well-established that CTCF mediates DNA-DNA interactions by forming dimers that directly bind to both sides of the $\mathrm{DNA}^{12,13}$, the regulatory mechanism controlling CTCF-DNA binding remains unknown. One hypothesis is that other factors such as cohesin play roles to mediate CTCF-DNA binding. Although multiple studies indicate that CTCF and cohesin interact with each other to contribute to chromatin structure maintenance ${ }^{6,14,15}$, a recent crystallographic structural study demonstrated that a specific CTCF mutation abolished CTCF-cohesin interaction, and further affected cohesin localization on CTCF sites, whereas CTCF binding on its sites remained unchanged ${ }^{16}$. This study indicates that CTCF recruits cohesin to its binding site, but CTCF-cohesin interaction does not regulate CTCF-DNA binding. So, to date it is not known which factor(s), if any, mediate CTCF-DNA binding to regulate chromatin interactions and gene expression.

The zinc finger protein 143 (ZNF143/ZFP143), was first discovered as selenocysteine tRNA gene transcription activating factor (STAF) in Xenopus laevis functioning as a sequence specific transcriptional activator of both Pol II and Pol III ${ }^{17,18}$. Later on, studies reveal that as a co-activator, ZNF143 might be a key regulator in T lymphoblastic leukemia (TLL) through its association with Notch $1^{19,20}$. Despite bioinformatic analysis suggesting that, similar to cohesin, ZNF143 co-localizes with $\mathrm{CTCF}^{7,21,22}$, and knocking down ZNF143 affects chromatin interaction at individual loci ${ }^{22}$, how CTCF and ZNF143 regulate the function of each other remains unknown. Building upon our previous work identify ZNF143 as an important regulator of Cebpa expression ${ }^{23}$, in this study, we used a hematopoietic model system to investigate whether ZNF143 mediates gene transcription through CTCF in a general manner.

\section{Results}

CTCF genome-wide DNA binding is dependent on ZNF143. Since ZNF143 and one of its family members, zinc finger protein 76 (ZNF76), share over $60 \%$ protein sequence identity and bind in vitro to the same DNA sequence ${ }^{17,23}$, whereas most commercial anti-ZNF143 antibodies recognize both ZNF143 and ZNF76 (data not shown), we first generated a specific antiZNF143 rabbit polyclonal antibody which only recognizes ZNF143, and not ZNF76, in both Western-blot and IP (Supplementary Fig. 1A, B). Then, we generated conditional Znf143 knockout mice to cross with Rosa26ERT2-cre mice so that Znf143 deletion can be induced by tamoxifen injection. After systematic time and dosage testing, a successful excision of $Z n f 143$ in adult hematopoietic stem and progenitor cells (HSPCs) was confirmed at the level of both RNA and protein (Supplementary Fig. 1C-G).
Under such conditions, we performed both ZNF143 and CTCF ChIP-seq in HSPCs. Surprisingly, we observed that, after Znf143 depletion, dramatic loss of the CTCF ChIP-seq signal was detected in regions where both ZNF143 and CTCF were enriched in wild type cells, without affecting CTCF mRNA or protein levels (Fig. 1a, Supplementary Fig. 2A, B; Supplementary Data 1). In contrast, no significant changes in CTCF localization were detected in regions where CTCF bound alone (Fig. 1a, Supplementary Data 1). Little is known regarding the regulation of CTCF loading onto chromatin by other factors, even though it is reported that ZNF143 and CTCF are both chromatin structure factors ${ }^{7,21,22}$. One possibility is that by forming a complex with CTCF, ZNF143 stabilizes the binding of CTCF on their cobinding regions. Indeed, reciprocal co-IP confirmed that ZNF143 and CTCF interacted with each other endogenously by immunoprecipitating either ZNF143 or CTCF (Fig. 1b). Furthermore, motif analysis using ZNF143 and CTCF ChIP-seq results from HSPCs found that ZNF143 and CTCF motifs were highly enriched within a $100 \mathrm{bp}$ distance in the mouse genome (Supplementary Fig. 2C, Supplementary Data 2). In contrast, an equal distribution within $\pm 2 \mathrm{~kb}$ regions was demonstrated with mutant ZNF143 motifs, which were randomly generated by Regulatory Sequence Analysis Tools (RSAT), a suite of bioinformatics tools to detect and analyze of cis-regulatory elements in genome sequences ${ }^{24}$ (Supplementary Fig. 2C). Surprisingly, deep analysis revealed that among 17,319 ZNF143 motifs located within $100 \mathrm{bp}$ from CTCF motifs, 10,544 ZNF143 motifs (60.88\%) were located 37 bp away from the nearest CTCF motifs (Fig. 1c, Supplementary Data 2). There is precedent for a TF being stabilized on its bound DNA by another factor bound nearby ${ }^{25}$, therefore the short distance between ZNF143 and CTCF binding motifs provided a hint about how ZNF143 regulated CTCF binding on their co-binding regions. Since it is known that the orientation of CTCF motifs is a key factor for chromatin loop formation $7,8,26,27$, we were wondering whether ZNF143 and CTCF motifs were oriented in a pattern. Four possible patterns exist between these two motifs as they are not palindromic: ZNF143 and CTCF motifs are 1) convergent on opposite strands (convergent); 2) divergent on opposite strands (divergent); 3) both on forward strand (F-F); 4) both on reverse strand (R-R). After analyzing 10,544 pairs of ZNF143 and CTCF motifs located $37 \mathrm{bp}$ away from each other, we observed $99.9 \%$ $(10,529)$ formed the convergent orientation (Fig. 1c, Supplementary Fig. 2C). With such a remarkably consistent pattern between ZNF143 and CTCF motifs across genome, we hypothesized that ZNF143 and CTCF work together genome-wide for loop formation and gene expression. On the other hand, we also queried whether CTCF facilitates ZNF143 binding. We observed higher enrichment of ZNF143 on ZNF143-CTCF shared sites compared to ZNF143-alone sites, suggesting that ZNF143 and CTCF regulate the binding of one another (Supplementary Fig. 2E). To further investigate which genomic regions ZNF143 and CTCF co-bound, ChIP-seq of ZNF143 and CTCF were further analyzed and a strong global correlation was detected, consistent with previous bioinformatic studies (Supplementary Fig. 2F-I $)^{21,22}$. Since approximately $90 \%$ of ZNF143 and CTCF co-bound regions were on promoter/enhancer regions (Supplementary Figs. 2D, G), we wondered whether ZNF143 is involved in CTCF-mediated transcriptional regulation. Active promoter scanning analysis confirmed that both ZNF143 and CTCF were highly enriched on active promoter regions compared to inactive promoter regions (Fig. 1d), and that ZNF143 was significantly enriched on CTCF-bound enhancer regions (Fig. 1e), indicating the co-localization of ZNF143 and CTCF on both promoter and enhancer regions. 

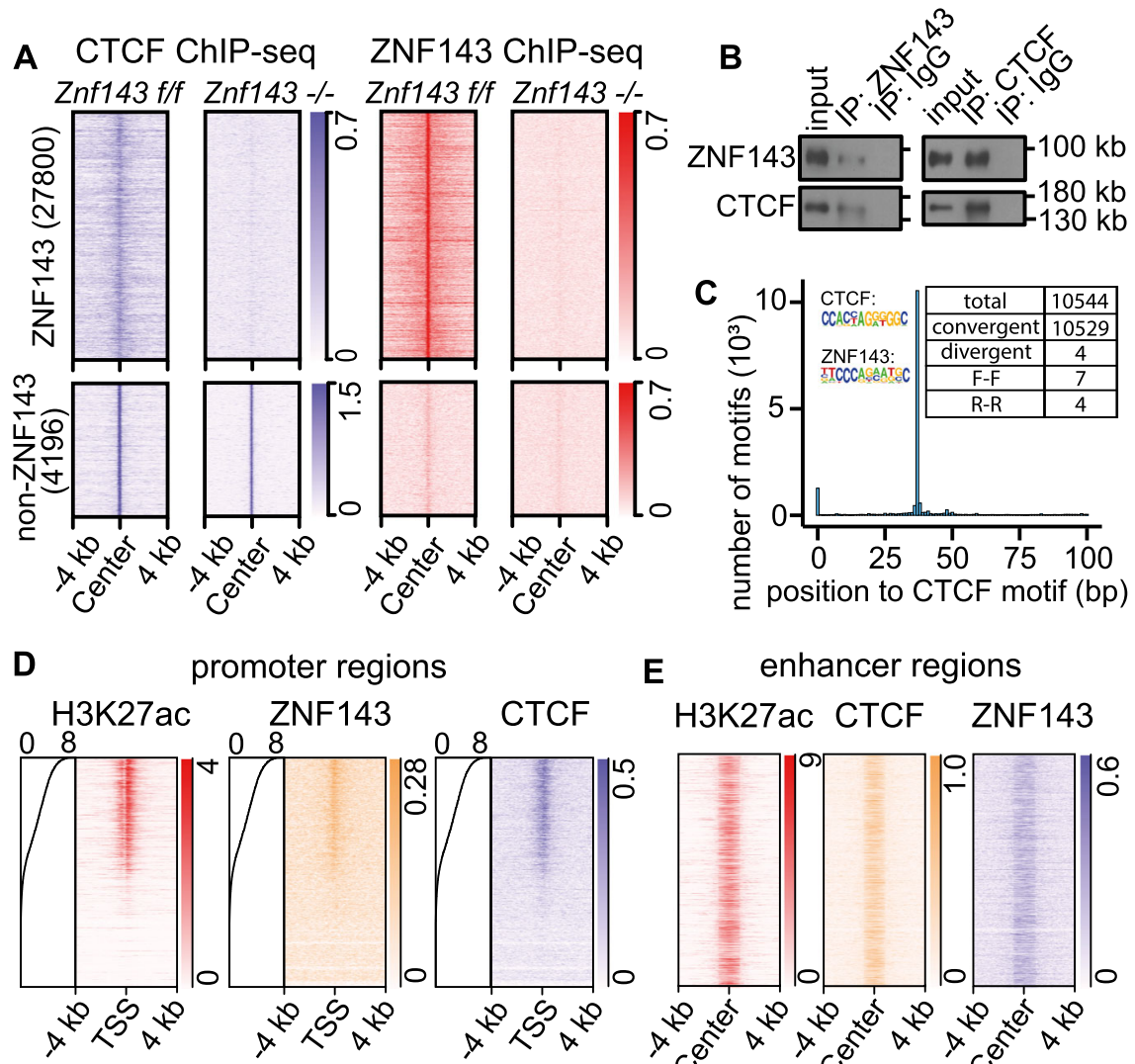

E enhancer regions

H3K27ac CTCF ZNF143

F ChIP-reChIP

G Global gene expression changes after Znf143 deletion
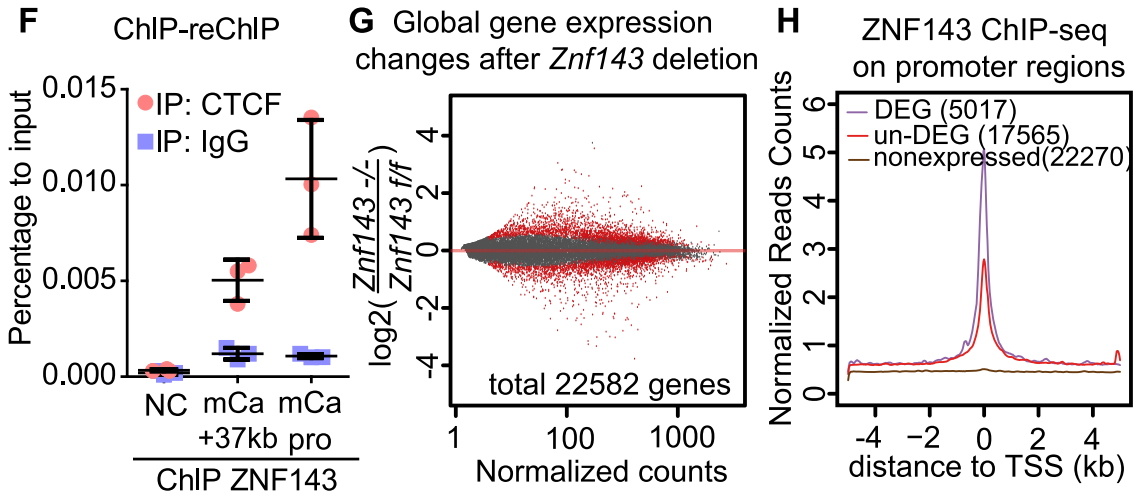

Fig. 1 ZNF143 stabilizes CTCF binding on promoter and enhancer regions to regulate gene transcription. a Heatmaps of ZNF143 and CTCF ChIP-seq in ZNF143 wild type (Znf143 f/f) and ZNF143 knockout (Znf143 - /-) murine HSPCs. b Reciprocal co-IP of ZNF143 and CTCF in murine HSPCs. Experiment is repeated independently for three times with similar results. c Histogram describing the distance between CTCF and ZNF143 motifs on the murine genome (mm10). The $X$-axis represents the relative distance between ZNF143 and CTCF motif, while the $Y$-axis represents the number of ZNF143 motifs in each bin. Each bin size is $1 \mathrm{bp}$. The table presents the number of ZNF143 motifs in the different orientation patterns to the nearest CTCF motifs within 10,544 ZNF143 motifs located 37 bp apart from the nearest CTCF motifs. d Heatmap of H3K27ac, ZNF143, and CTCF ChIP-seq in Znf143 f/f murine HSPCs within the regions $\pm 4 \mathrm{~kb}$ from the transcription start site (TSS). Gene order is ranked by RNA expression from high to low in Znf143 f/f cells (heatmap from top to bottom). e Heatmap of H3K27ac, ZNF143, and CTCF ChIP-seq in Znf143 f/f murine HSPCs within \pm 4 kb from CTCF-bound enhancer regions. f ChIP-reChIP of ZNF143 and CTCF on a negative intergenic control region (NC), Cebpa promoter region ( $\mathrm{mCa}$ pro), and Cebpa $+37 \mathrm{~kb}$ enhancer region ( $\mathrm{mCa}+37 \mathrm{~kb}$ ) in Znf143 f/f murine HSPCs. Y-axis: the mean \pm SD from triplicate experiments. g MA plot demonstrating gene expression changes between $Z n f 143 f / f$ and ZNF143 -/- murine HSCs. Red dots represent differentially expressed genes $(p<0.05) . n=$ three biological replicates. $P$-value is determined by the Wald test. $\mathbf{h}$ The mean plot of ZNF143 ChIP-seq within $\pm 4 \mathrm{~kb}$ TSS regions in Znf143 f/f murine HSPCs. Genes are divided into (1) differentially expressed genes (DEG), (2) non-differentially expressed genes (un-DEG), and 3) non-expressed genes (non-expressed) as defined in (g). HSPCs: c-kit+, lineage- cells; HSCs: LSK+ (lineage-, Sca-1+, c-kit+), CD150+, CD48- cells. ChIP-seq in panels (a), (c), (d), (e), and (h) was performed in two biological replicates. Result was shown in one replicate.

To address the limitation of ChIP-seq, whose resolution is modest and therefore apparent co-localization may not necessarily indicate close association or interaction, we performed ChIP-reChIP for further validation. Consistent with our hypothesis, enrichment on promoter and enhancer regions were detected in CTCF pull-down but not IgG control in the elute from ZNF143 ChIP (Fig. 1f). Together with our co-IP results (Fig. 1b), it demonstrated that ZNF143 and CTCF form a complex to bind to both promoter and enhancer regions. Furthermore, over 5,000 genes displayed significant expression 
changes after Znf143 deletion, corresponding with high ZNF143 ChIP-seq signals detected on their promoter regions (Fig. 1g, h, Supplementary Data 3). Taken together, our results indicate that ZNF143 is a critical mediator for CTCF-involved gene transcription by regulating CTCF binding on promoter and enhancer regions.

ZNF143 depletion affects CTCF-bound promoter-enhancer loop intensities while compartments and TADs remain unchanged. Next, we investigated whether CTCF-bound chromatin-chromatin interactions were disrupted after ZNF143 depletion by performing in situ $\mathrm{HiC}$ using $Z n f 143 \mathrm{f} / \mathrm{f}$ and $Z n f 143$-/- HSPCs. Within the 11,930 loops detected in Znf143 f/f cells at $5 \mathrm{~kb}$ resolution, 4017 loops significantly decreased in Znf143 -/- cells. Further annotation of these affected loops revealed that $38 \%$ of them mediated promoter-enhancer and $10.7 \%$ mediate promoter-promoter interaction, suggesting that the loss of ZNF143 mainly affected promoter-enhancer loops (Fig. 2a, b, Supplementary Data 4,5 ). One alternative possibility is that the loss of ZNF143 affects the expression of some other chromatin structure factor, leading to indirect changes of promoter-enhancer loops. To exclude this possibility, after dividing loops into (1) differentially changed and (2) unchanged groups, CTCF motif scanning was performed on their anchors, followed by ZNF143 ChIP-seq analysis on regions $\pm 2 \mathrm{~kb}$ from these motifs (Supplementary Data 6). The results demonstrated that consistent with loop intensity changing, ZNF143 was highly enriched around these motif regions located on differential loop anchors, compared to anchors of unchanged loops (Fig. 2c, d). In line with these findings, we also detected significantly decreased CTCF binding on ZNF143-dependent loop anchors, suggesting that ZNF143 directly participated in the maintenance of CTCF-involved promoter-enhancer loops (Fig. 2e, Supplementary Fig. 3A, B).

Since our previous study revealed that ZNF143 is important for Cebpa regulation ${ }^{23}$, and that ZNF143 and CTCF were detected on both the Cebpa promoter and a previously reported $+37 \mathrm{~kb}$ enhancer region (DRE $37 \mathrm{~kb}$ region) (Fig. 1f), the Cebpa locus was selected for further validation. By performing chromosome conformation capture $(3 \mathrm{C})$, we confirmed the decrease of interaction frequency between the Cebpa promoter and the 37 $\mathrm{kb}$ Cebpa enhancer after deletion of ZNF143 (Fig. 2f). In agreement with the loop change, significant down-regulation of Cebpa mRNA levels and decreases of ZNF143 and CTCF enrichment on both the Cebpa promoter and enhancer were detected (Fig. 2g, h), indicating that loss of ZNF143 affected CTCF binding on both the Cebpa promoter and enhancer, leading to down-regulation of Cebpa expression by decreasing loop frequency. Taken together with our other results, we conclude that ZNF143 mediates CTCF binding on chromatin to regulate promoter-enhancer loops, thus mediating gene transcription.

Since the majority of ZNF143 binding occurred in promoter regions (Supplementary Fig. 2D, F, G), we asked whether ZNF143 was only involved in CTCF-bound promoter-enhancer loops, but not large-scale chromatin structures such as compartmentalization and TAD formation. Indeed, we did not observe significant changes in compartmentalization (Fig. 3a, Supplementary Fig. 4A). Moreover, genome-wide contact-probability scanning revealed no difference between $Z n f 143 \mathrm{f} / \mathrm{f}$ and Znf143 -/HSPCs (Fig. 3b). We conclude that loss of ZNF143 does not affect the global distribution of active and inactive chromatin. Next, we zoomed in to analyze TAD formation. TADs are detected with a pair of strong boundaries, or insulation scores, and typically span a distance from approximately $100 \mathrm{~kb}$ to $1 \mathrm{Mb}$ in murine and human genomes. They are characterized by the feature that two loci within the same TAD interact with each other more frequently than two loci in different TADs ${ }^{4,5}$. Using the bioinformatic tool Arrowhead to define $\mathrm{TADs}^{7}$, we detected 3,086 TADs in Znf143 f/f HSPCs and 3,114 TADs in Znf143 -/(Supplementary Fig. 4B, Supplementary Data 7), indicating that loss of ZNF143 did not affect TAD numbers. Furthermore, no significant changes in TADs were detected globally (Fig. 3c, Supplementary Fig. 4C). Insulation score analysis was also performed to double confirm no change in TADs formation (Fig. 3d $)^{28}$. In line with that observation, 2,331 CTCF-alone peaks and less than 400 CTCF-ZNF143 shared peaks were detected on TAD boundaries (Supplementary Fig. 4D), indicating that ZNF143 is a novel factor mediating CTCF-bound loops but not TADs.

ZNF143 is essential for hematopoietic stem and progenitor cells integrity. Considering the genome-wide regulation of CTCF-bound promoter-enhancer loops and gene expression, we were interested in assessing the biological effects of loss of Znf143, using the hematopoietic system as a model. As we observed a reduction in HSPC populations at a late time point after the deletion of Znf143 (Supplementary Fig. 1D), we next performed systematic analysis to examine the biological effects of loss of Znf143 in hematopoiesis. The Vav1-iCre mouse model is widely used for studies of hematopoiesis in the embryonic stage as it starts expressing at E9.5 and is greatly enhanced at E12.5 in hematopoietic cells ${ }^{29}$. We crossed Znf143 flox/flox mice with Vav1-iCre mice to investigate the phenotype in hematopoiesis during embryonic development. In line with much paler embryos at E14.5, smaller and paler Znf143 -/- fetal livers were observed, consistent with a significant decrease in cell counts from whole fetal livers (Fig. 4a, b). Furthermore, no Znf143 -/mice were found among 65 viable progenies (Supplementary Fig. 5A), indicating that loss of $Z n f 143$ induced a severe embryonic anemia and lethality. Next, the role of ZNF143 in adult hematopoiesis was examined by crossing Znf143 flox/flox mice with Mx1-Cre mice for inducible deletion through poly I:C injection, and reduction of ZNF143 protein was detected in HSPCs 8 days after the first poly I:C injection (Supplementary Fig. 1G). After inducible deletion of $Z n f 143$, detection of a significant reduction in white blood cell counts indicated its critical role in adult hematopoiesis (Supplementary Fig. 5B). To systematically investigate which subpopulations were affected by the loss of ZNF143, multiple stem cell and progenitor cell populations were profiled after Znf143 excision using both fetal liver cells and total bone marrow cells (BMCs). Consistent results obtained using both embryonic and adult hematopoietic cells demonstrated that long-term hematopoietic stem cells (LTHSCs), short-term hematopoietic stem cells (ST-HSCs), multipotent progenitor cells (MPPs), common myeloid progenitor cells (CMPs), granulocyte progenitor cells (GMPs), and megakaryocyte-erythroid progenitor cells (MEPs) were all dramatically reduced after Znf143 excision (Fig. 4c, d, Supplementary Fig. 5C, D), suggesting the key role of ZNF143 in the maintenance of the integrity of HSPCs. To exclude the potential hematopoietic cell-extrinsic effects of loss of $Z n f 143$, competitive transplantation was performed using adult bone marrow cells from $Z n f 143 \mathrm{f} / \mathrm{f}$, Mx1-Cre + or Znf143 $\mathrm{f} / \mathrm{f}$ mice. After detection of successful engraftment, $Z n f 143$ excision was induced by poly I:C injection, followed by analysis of peripheral blood (PB) chimerism over 4 months. In agreement with the results obtained with fetal liver cell transplantation (Supplementary Figs. 5E, F, 6 ), continuous reduction of engraftment was observed after deletion of $Z n f 143$, whereas wild type cells succeeded in reconstituting the hematopoietic system over 4 months (Fig. 4e, f). 


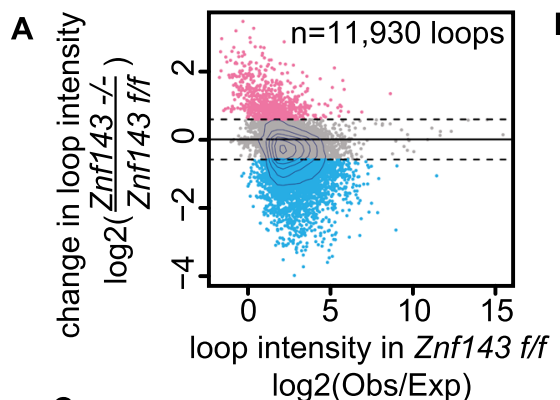
B Loop changes in Znf143 -/- cells

$$
6.44 \% \mathrm{n}=11,930
$$
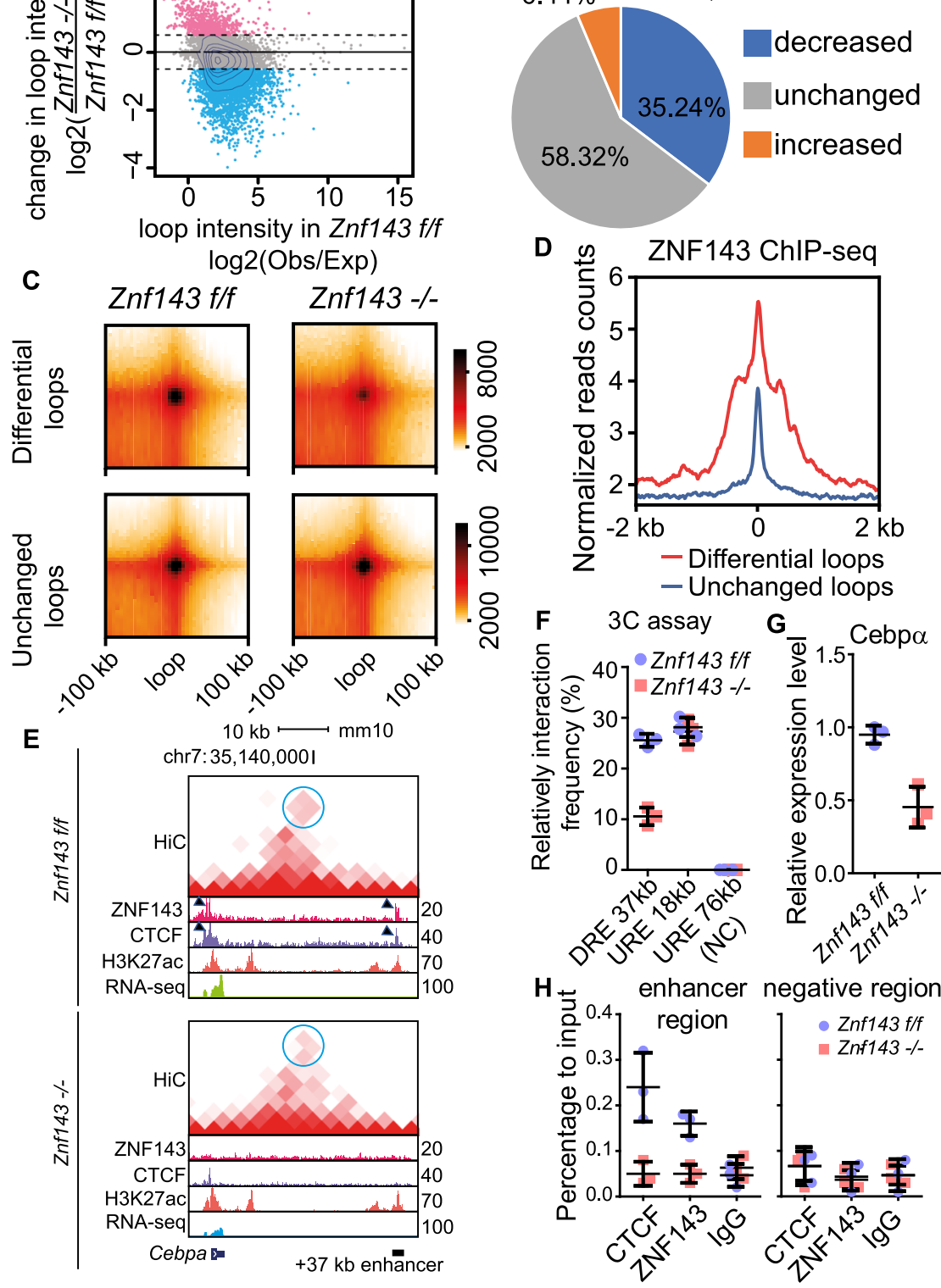

Fig. 2 Promoter-enhancer loops are maintained by ZNF143. a Density plot describes individual intensity change of loops detected in $Z n f 143 f / f$ murine HSPCs (LSK+) after deletion of Znf143. Red dots present loops with increased intensity (1.5-fold threshold, 768 loops), while blue dots present loops with decreased intensity (-1.5-fold threshold, 4204 loops). b Pie chart describes a statistical summary of loop changes using the same categories described in (a). c Aggregate peak analysis for intensity of differential loops. Loops divided into different groups as described in (a) are aggregated at the center of a $100 \mathrm{~kb}$ window in $5 \mathrm{~kb}$ resolution. d Mean plot of ZNF143 ChIP-seq within $\pm 2 \mathrm{~kb}$ from CTCF motifs on loop anchors. Loops are divided into different groups as described in (a). $n=$ two biological replicates. e UCSC genome browser shot presents HiC interaction frequencies, ChIP-seq profiles of ZNF143, CTCF, and H3K27ac, and RNA expression on Cebpa locus in both Znf143 f/f and Znf143 - / - murine HSPCs. The balanced HiC two-dimensional contact matrix is plotted on top. The color intensity presents interaction frequency, while blue circles indicate the Cebpa 37 kb enhancer-promoter loop. ZNF143 and CTCF binding profile are presented below, while H3K27ac histone mark indicates an active chromatin status. Dark triangles indicate ZNF143 and CTCF peaks located on loop anchors. The maximum $Y$-axis value of ChIP-seq signal is set as indicated. $\mathbf{f}$ The interaction frequencies between the Cebpa promoter and the Cebpa $+37 \mathrm{~kb}$ enhancer ("DRE $37 \mathrm{~kb}$ ") or with a non-related control region located $18 \mathrm{~kb}$ upstream from the Cebpa promoter ("URE $18 \mathrm{~kb}$ ") were examined by $3 \mathrm{C}$ qPCR in both Znf143 f/f and Znf143 - /- murine HSPCs. The $76 \mathrm{~kb}$ upstream region from the Cebpa promoter (URE $76 \mathrm{~kb}$ ) is presented as a negative control region (NC). g Dot plot describes relative Cebpa mRNA levels in Znf143 f/f and Znf143 -/- murine HSCs (LSK+, CD150+, CD48-) normalized to $\beta$-actin. h ChIP-qPCR of ZNF143 and CTCF on the Cebpa $+37 \mathrm{~kb}$ enhancer region or intergenic negative control region. All dot plots are presented in mean \pm SD from three biological replicates experiment.

These results indicated that ZNF143 is a key cell-intrinsic factor maintaining HSPC integrity.

ZNF143-CTCF loops regulate pathways critical for hematopoiesis. Finally, we sought to identify the genes and pathways regulated by ZNF143-CTCF loops contributing to the murine phenotype. By integrating gene expression and ZNF143 localization on promoter regions, we narrowed the number of highconfidence ZNF143 direct target genes down to 1467 (Supplementary Data 8). We observed higher global expression levels 

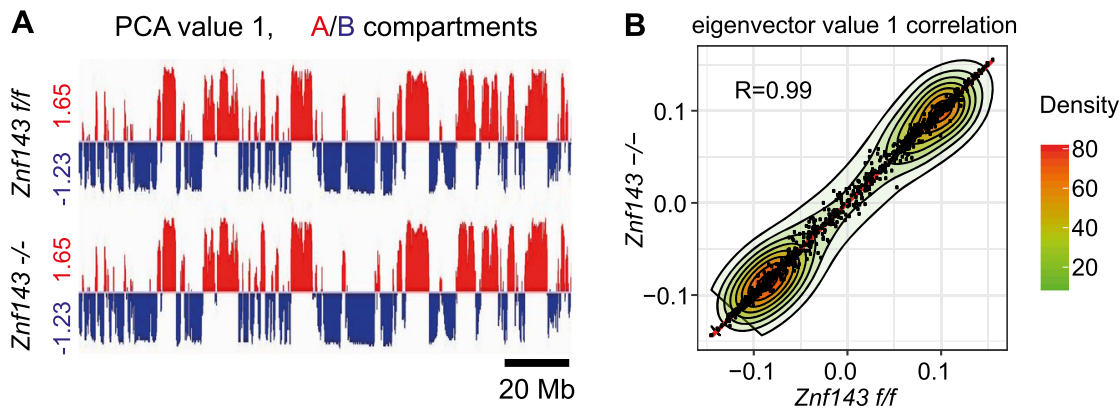

C

Znf143 f/f

Znf143 -/-
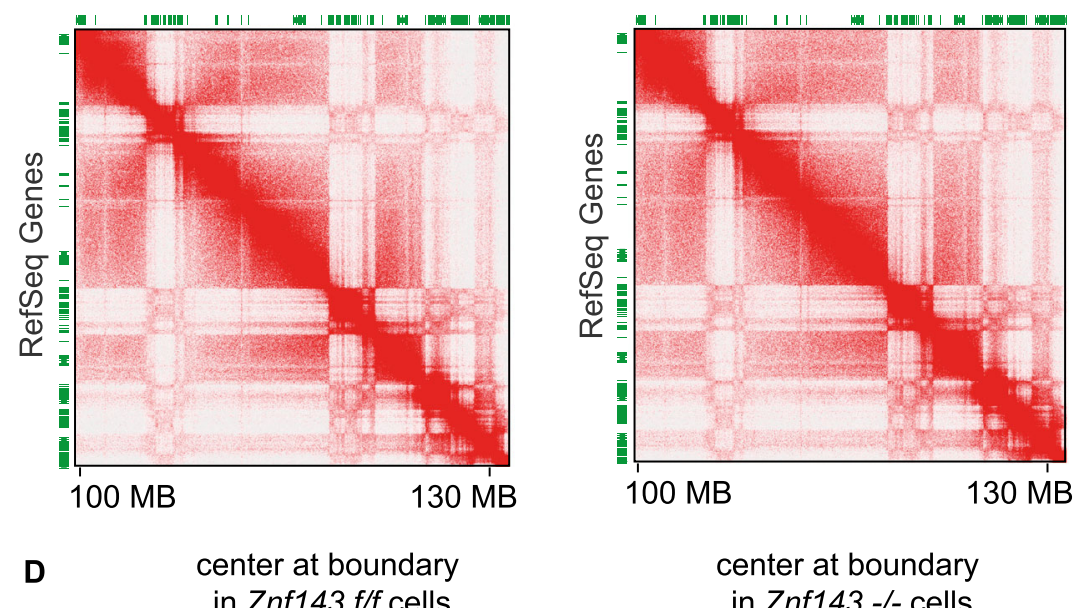

center at boundary in Znf143 -/- cells

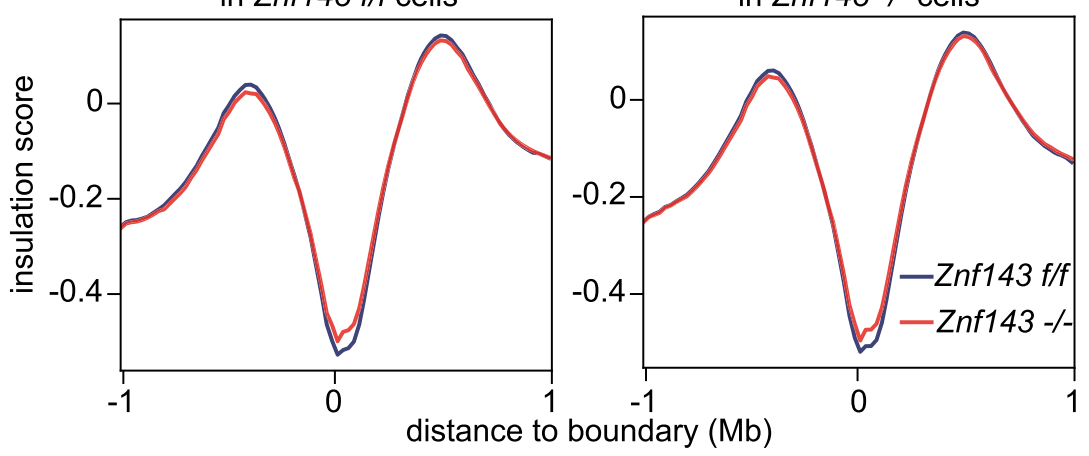

Fig. 3 Chromosome compartments and TADs mainly remain unchanged after Znf143 deletion. a PCA analysis for the first principle component representing compartment $A$ and $B$ on chr1 at $50 \mathrm{~kb}$ resolution. b Density plot describes global correlation between the eigenvector value from wild type ( $Z n f 143 f / f)$ and Znf143 depleted (Znf143 -/-) LSKs at $1 \mathrm{Mb}$ resolution. The $X$-axis represents the eigenvector value in $Z n f 143 \mathrm{f} / f$ cells on each genome locus, while the $Y$-axis the value in Znf143 -/ - cells in the same locus. c Comparison of TADs in wild type (Znf143 f/f) and Znf143 knockout (Znf143 - /-) cells. A snapshot of balanced contact matrices on chr1:100-130 Mb. d Mean plot describes genome-wide insulation score around TADs boundaries. The $X$ axis represents the genome distance to the TADs boundaries, while the $Y$-axis the insulation score. The 0 position on the $X$-axis in the left panel indicates TAD boundaries in Znf143 f/f cells, while in the right panel TAD boundaries in Znf143 - / - cells.

among these ZNF143 direct targets compared to all other genes, and these ZNF143 targets were also significantly downregulated after deletion of Znf143 (Fig. 5a). Principal component analysis (PCA) indicated different clustering between wild type and Znf143 deleted cells (Supplementary Fig. 7A, B). Furthermore, multiple pathways (such as ribosomal pathways, metabolismrelated pathways, and hematopoietic stem cell-related pathways) and genes critical for hematopoiesis (RUNX1 and Gata2, among others) were downregulated after the loss of Znf143 (Fig. 5b, Supplementary Fig. 7C-E). These genes were reported as key regulators of hematopoietic stem cells, and mutations of these genes and disorders of these pathways are commonly observed in bone marrow failure, a disease syndrome defined as a drastic decline in the ability of bone marrow to produce mature blood ${ }^{30,31}$. Consistent with the observation of dramatic disappearance of mature blood cells after excision of Znf143 (Fig. 4a, b, Supplementary Fig. 5B), our data suggested that ZNF143 maintained HSPCs integrity by regulating key genes and pathways. Furthermore, after integrating global loops and CTCF binding, we confirmed a significant reduction of CTCF binding on the promoter-enhancer loop anchors on ZNF143 regulated genes, consistent with decreased loop intensities (Fig. 5c, d, Supplementary Fig. 7F). Together, these results indicate that ZNF143-CTCF-mediated promoter-enhancer loops play important roles to regulate key genes and pathways essential for the maintenance of HSPC integrity. 


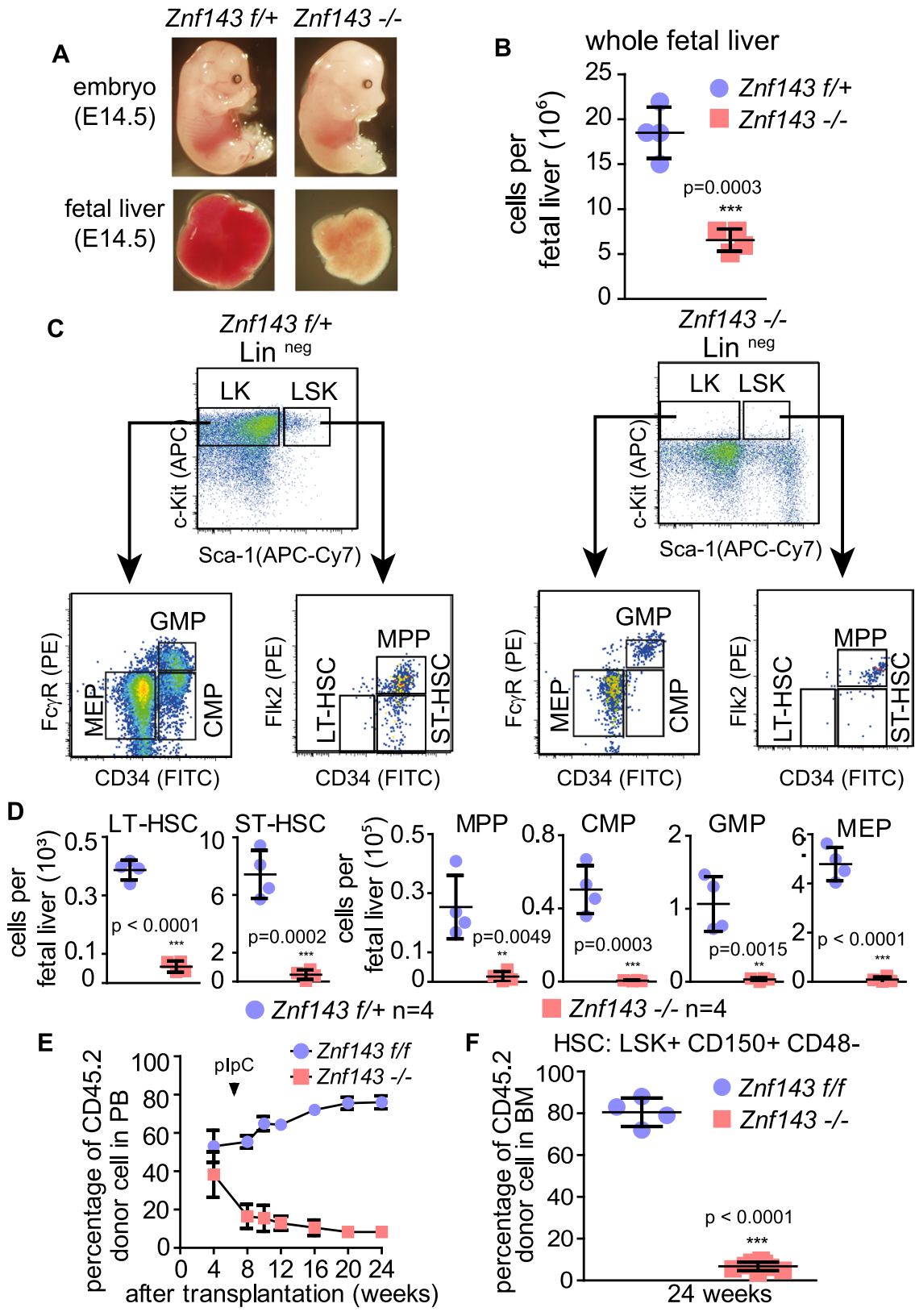

Fig. 4 ZNF143 is essential for maintenance of HSPC integrity. a Image of embryos and fetal livers of Znf143 f/ + and Znf143 conditional knockout ( $Z n f 143$ -/-) mice at embryonic day 14.5 (E-14.5). The floxed Znf143 allele was deleted specifically in hematopoietic cells by crossing with Vav1-iCre mice. b Dot plot describes absolute numbers of nucleated cells in Znf143 f/+ and Znf143 -/- fetal liver at E-14.5 ( $n=4$ in each group). Values are presented as mean \pm SD. c Dot plot of flow cytometry analysis of different HSPC populations in Znf143 f/ + and Znf143 - / fetal liver cells at E-14.5. The upper left panel demonstrates the LSK cells (Lin- c-Kit+ Sca-1+) or LK cells (Lin- c-Kit+) which were further analyzed in the lower-left panel. d Absolute numbers of HSPCs as defined in (c), including LT-HSC (LSK CD34- Flk2-), ST-HSC (LSK CD34+Flk2-), MPP (LSK CD34+Flk2+), CMP (LK CD34+FcyR-), GMP (LK CD34+FcyR+), and MEP (LK CD34- FcyR-), comparing fetal liver cells from Znf143 f/+ with Znf143 -/- embryos. Data were obtained from four pairs of littermates. Values are presented as mean \pm SD. e Donor chimerism in peripheral blood (PB) for 24 weeks after transplantation with Znf143 $f / f$ or Znf143 f/f, Mx1-Cre+ whole bone marrow cells after removal of red blood cells. Znf143 was deleted by plpC injection for three consecutive days 2 weeks after transplantation. Donor chimerism was monitored by measuring the percentage of CD45.2 positive cells in the peripheral blood at the indicated time points. Znf143 $f / f: n=4, Z n f 143-/-: n=8$. Values are presented as mean \pm SD. $\mathbf{f}$ Dot plot demonstrating the donor chimerism of HSCs (CD150+CD48LSK+) in bone marrow (BM) 24 weeks after transplantation. Each dot represents the percentage of CD45.2 positive HSCs in one recipient mouse. Znf143 $f / f: n=4, Z n f 143-/-: n=8$. Values are presented as mean \pm SD. $P$ values in $(\mathbf{b}),(\mathbf{d})$, and (f) are determined by the two tailed unpaired $t$ test $\left({ }^{*} p<0.05\right.$, ${ }^{\star *} p<0.01,{ }^{\star \star *} p<0.001$ ).

\section{Discussion}

As a key factor in the maintenance of chromatin structure, ubiquitously expressed in most organs and highly conserved in higher eukaryotes, CTCF is critical for multiple cellular processes. In addition to resulting in disruption of blastocyst stage processing and exhibiting early embryonic lethality, deletion of CTCF also results in defects of lineage differentiation ${ }^{32,33}$. Although it is widely reported that CTCF and other factors such as cohesin co-localize together to mediate chromatin-chromatin interactions, a recent study of the crystallographic structure of the 

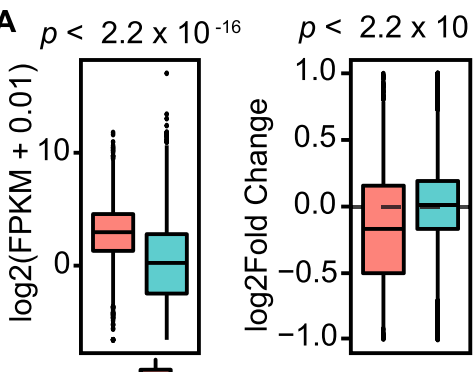

追 ZNF143 (n=1467)

追 other $(n=21649)$

C

ZNF143 related

Znf143 f/f Znf143 -/-

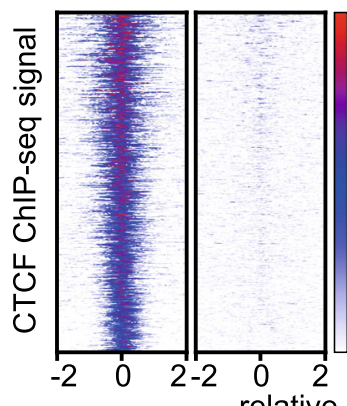

relative distance $(\mathrm{kb})$

D
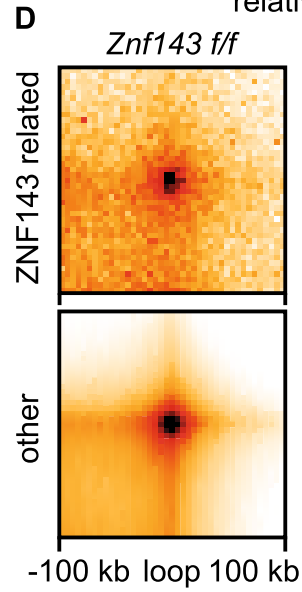

\footnotetext{
-100 kb loop $100 \mathrm{~kb}-100 \mathrm{~kb}$ loop $100 \mathrm{~kb}$
}

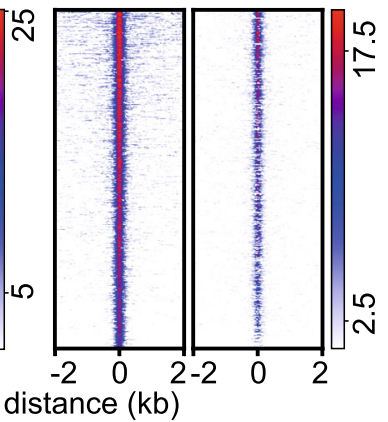

B Down-regulated pathways

translation -

transcriptional activity. of smad heterotrimer transcription cholesterol biosynthesis p53 regulation glycolysis gluconeogenesis DNA damage by gamma radiation ribosomecalcium signalinghematopoiesis stem cell long term

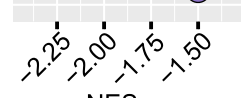

NES

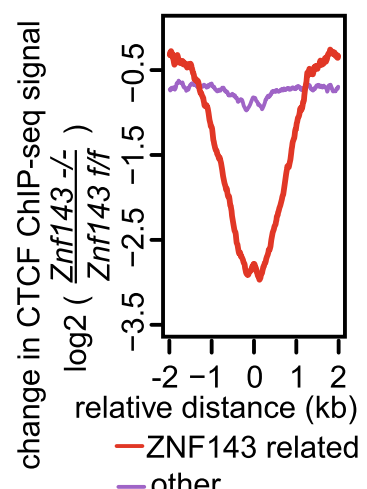
- other

\section{E with ZNF143}

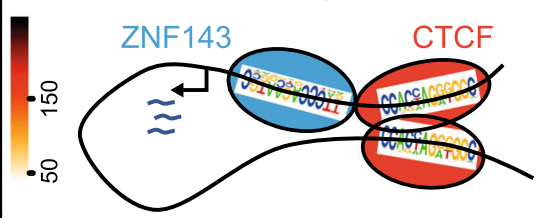

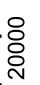

Fig. 5 ZNF143-CTCF-bound loops regulate key genes and pathways contributing to the stem cell phenotype. a Left panel, box plot describes log2 fragment per kb per million (FPKM) of genes in Znf143 f/f HSCs that are 1) highly enriched of ZNF143 on their promoters (ZNF143, 1467 genes) or 2) not associated with high ZNF143 enrichment (other, 21,649 genes). Right panel, log2 fold change in expression of genes grouped as in left panel. $P$ values determined by the two-sided Mann Whitney Wilcoxon Test. $n=$ three biological replicates. Boxplot represents median and the $25^{\text {th }}$ and $75^{\text {th }}$ percentiles of the distribution. The upper and lower whisker represents data no further than 1.5 times the IQR from 25th or 75th percentiles. $\mathbf{b}$ Bubble plot describes downregulated pathways after Znf143 excision discovered by Gene Set Enrichment Analysis (GSEA). The X-axis represents normalized enrichment score (NES), while bubble size represents normalized $p$-value (transformed into -log10 format). P-value is determined as described previously 52 . c Left panel, heatmap of the CTCF ChIP-seq signal within $\pm 2 \mathrm{~kb}$ from CTCF motifs on loop anchors. Each column represents the relative distance to the CTCF motif, while each row represents normalized read counts of CTCF on the loop anchor region. Loops are divided into two groups according to ZNF143 binding on their anchors. The right panel demonstrates the mean plot of the average changes of the CTCF ChIP-seq signal within $\pm 2 \mathrm{~kb}$ from CTCF motifs on loop

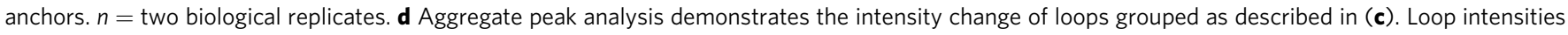
are aggregated at the center of a $100 \mathrm{~kb}$ window in $5 \mathrm{~kb}$ resolution. e Proposed model depicting regulation of CTCF-bound loops by ZNF143.

CTCF-cohesin interaction demonstrated that this interaction does not regulate CTCF-DNA binding ${ }^{4-8,14,16}$.

A previous study suggested that zinc finger TF ZNF143 is involved in promoter-enhancer loops ${ }^{22}$; however, how it regulates such loops is unknown. In this study, we further investigate the function between ZNF143 and CTCF and demonstrate that ZNF143 is a key regulator of CTCF-DNA binding across the genome and as a result mediates CTCF-bound promoter-enhancer loops. More than colocalizing on active promoter and enhancer regions in the mouse genome, analysis 10,544 ZNF143 motifs suggested a specific spacing of $37 \mathrm{bp}$ between a ZNF143 and CTCF binding sites. Such a specific distance between two different TF binding motifs across the genome has been rarely reported, which led to the hypothesis that ZNF143 served as a regulator of CTCF by mediating its ability to bind to DNA. Loss of ZNF143 led to the loss of CTCF-DNA binding on these loci, mainly affecting CTCFbound promoter-enhancer loops, but not TAD formation or 
compartmentalization, consistent with global changes in gene expressions. Considering the ubiquitous expression and high homology of both ZNF143 and CTCF among mammals, we conclude that ZNF143 is a novel CTCF regulator that mediates CTCF-DNA binding on promoter and enhancer regions to form promoter-enhancer loops. However, we also noticed that a portion of affected loops are not associated with promoter regions (Supplementary Data 5), suggesting that ZNF143 might mediate other types of loops bound by CTCF, as CTCF is well known to play a critical role on insulators ${ }^{34,35}$.

It is quite interesting that ZNF143 mainly regulates CTCFbound promoter-enhancer loops but not TADs. That loss of ZNF143 only affects CTCF-bound promoter-enhancer loops suggests a novel subgroup of CTCF sites, regulated by ZNF143 and restricted to promoter and enhancer regions. It is widely reported that CTCF and cohesin are the major factors to maintain TAD formation across different cell types ${ }^{4-9,15,16,21,26,36,37}$. However, in contrast to conservation of TADs in different cell types, loop formations are far more dynamic and regulated not only by CTCF and cohesin, but other factors such as $\mathrm{YY} 1^{11}$ and chromatin remodeler Brg1 ${ }^{38}$, among others. Recent studies using high-resolution Micro-C revealed a large portion of promoter-enhancer loops under $200 \mathrm{bp}$ resolution ${ }^{39,40}$. Interestingly, these new discovered promoter-enhancer interactions were often detected in the absence of CTCF and cohesin occupancy. Although their biological function remains to be explored, this finding suggests that further studies are required to better understand genome organization and its regulation. Our results in this study strongly suggest that ZNF143 mainly regulates finescale genome organization without altering higher-order chromatin structure.

Another interesting aspect of future study is regarding ZNF143 and CTCF motif distance and orientation. Since it has not been reported previously that the ZNF143 motifs lie precisely $37 \mathrm{bp}$ from the nearest CTCF motif, it will be of interest in subsequent studies to investigate how this $37 \mathrm{bp}$ distance mediates their colocalization. The DNA binding affinity of one TF can be affected by another factor binding nearby ${ }^{25}$, so one hypothesis is that ZNF143 stabilizes CTCF-DNA binding when located 37 bp away. Another possibility is that ZNF143 acts as a pioneer factor to remodel chromatin status to make CTCF sites located $37 \mathrm{bp}$ away accessible. Future experiments will reveal which hypothesis, or both, can account for the dependency of CTCF-DNA binding, and promoter-enhancer interactions, on ZNF143. On the other hand, in line with that CTCF motif orientation is critical for loop formation, how ZNF143 motif orientation contributes to looping will be of interest, as almost all ZNF143 and CTCF motifs located $37 \mathrm{bp}$ apart from each other form the convergent orientation. It will be of interest to investigate whether changes in ZNF143 motif orientation will affect nearby CTCF binding, and hence affect looping. Of note, we do not have a clear answer on whether ZNF143 motifs are required on both anchors, and therefore additional studies with more detailed analysis will be necessary to address this question.

How chromatin organization mediates hematopoietic stem and progenitor cell integrity is an important aspect yet not fully explored. Previous studies found that complete loss of SMC3, one of the core cohesin ring components, caused bone marrow aplasia due to abnormal chromatid segregation, whereas Smc3 haploinsufficiency increased self-renewal in culture and in vivo ${ }^{41}$. On the other hand, deletion of cohesin binding component STAG2 but not STAG1 increased HSC self-renewal and reduced differentiation capacity, suggesting that the cohesin complex plays different roles in HSPCs driven by different STAG subunits ${ }^{42}$. Other than the cohesin complex, coactivators and mediators were also reported to be critical for HSPC function through facilitating active histone marks, such as $\mathrm{H} 2 \mathrm{~A} . \mathrm{Z} / \mathrm{H} 3 \mathrm{~K} 27$ acetylation on promoter/enhancer regions ${ }^{43-45}$. Although our studies of ZNF143 reveal another aspect of the regulation of HSPC chromatin organization through mediating the integrity of CTCFbound loops, it is not clear that whether ZNF143 is critical for all HSPCs or only in LT-HSCs. As homo- or hetero-dimers could be formed within zinc- coordinating TFs, we anticipate that additional TFs will be discovered to contribute to chromatin structure and hence regulate HSPC integrity.

The model that ZNF143 mediates CTCF-bound promoterenhancer loops may have relevance to interpreting previous studies. For example, it is reported that ZNF143 co-binds to approximately $40 \%$ of Notch 1 sites in TLL ${ }^{19,20}$. Considering that Notch1 gain-of-function mutations are mostly observed in TLL patients, this might represent another example of CTCF dependency on another TF. Since disruption of this ZNF143-CTCF regulatory axis results in a severe anemia phenotype in both embryos and adults, in the agreement of ubiquitous expression and high homology of both ZNF143 and CTCF among mammals, the regulation of CTCF-bound loops controlled by ZNF143 might contribute to multiple physiologic and disease processes.

\section{Methods}

Mice. C57BL/6J, Mx1-Cre ${ }^{46}$, and Vav1-iCre mice ${ }^{29}$ were obtained from The Jackson Laboratory; Rosa26ERT2-Cre mice ${ }^{47}$ were obtained from Yoshiaki Ito. $Z n f 143$ flox mice were generated by inserting loxP site before and after exon 1 of Znf143. Znf143 f/f mice were crossed with Rosa26ERT2-Cre+ mice to generate Znf143 f/f x Rosa26ERT2-Cre+ and Znf143 f/f x Rosa26ERT2-Cre- mice. Then Znf143 deletion was achieved with tamoxifen injection as described in Figure S2. Znf143 f/f x Vav1-iCre- male mice were crossed with Znf143 f/+ x Vav1$i C r e+$ female mice to avoid germline deletion of the floxed allele. To study the function of Znf143 in adult hematopoiesis, Znf143 f/f mice were crossed with $M x 1$ Cre+ mice to generate $Z n f 143 \mathrm{f} / \mathrm{f} \times \mathrm{M} \times 1$-Cre+ and $Z n f 143 \mathrm{f} / \mathrm{f} \times \mathrm{M} \times 1$-Cre- mice. Littermates of Znfl43 f/f x Mx1-Cre+ and Znfl 143 f/f; Mxl-Cre- mice were injected intraperitoneally (i.p.) with $300 \mu \mathrm{g}$ poly I:C for three consecutive days. Then mice were euthanized at day 8 after the first injection to assess deletion. All mice were housed in a sterile barrier facility within the Comparative Medicine facility at the National University of Singapore under housing condition of $22^{\circ} \mathrm{C}$ temperature, $50 \%$ humidity and a 12:12 light/dark cycle. All mice experiments performed in this study were approved by Institutional Animal Care and Use Committee of National University of Singapore. In this study, 8- to 12-week-old male mice were used bone marrow harvesting or transplantation; 12- to 16-week-old female mice were used for time mating. See "Methods" for more information.

Antibodies and primers. All antibodies and primers used in this study were listed in supplementary data 10 and 11. All full scans of Western-blot are provided in Supplementary Fig. 8.

Fetal liver cell isolation. For fetal liver isolation, $Z n f 143 \mathrm{f} /+\mathrm{x}$ Vav1-iCre + male mice were mated with $Z n f 143 \mathrm{f} / \mathrm{f}$ female mice in the evening on day 0 . Embryos were collected at E14.5-day stage for fetal liver isolation and genotyping. Fetal livers were kept in PBS at $4{ }^{\circ} \mathrm{C}$ until genotyping of each embryo was completed. Fetal livers from Znf143 f/+ x Vav1-iCre- (Znf143 f/+) and Znf143 f/f x Vav1-iCre+ (Znf143 -/-) embryos were dissociated into single cells by passing through a 40 $\mu \mathrm{m} \mathrm{BD}$ strainer with a $1 \mathrm{ml}$ syringe plunger in PBS plus $2 \%$ FBS and $2 \mathrm{mM}$ EDTA.

Bone marrow cells (BMCs) isolation. After $\mathrm{CO}_{2}$ euthanasia, mice were dissected for vertebrae, femur, tibia, and hip collection. Bones were crushed using a pestle with ice-cold PBS and then bone marrow cells suspension were filtered through a $70 \mu \mathrm{m} \mathrm{BD}$ cell strainer. Red blood cells were removed with RBC lysis buffer treatment. After filtering, a $20 \mu \mathrm{l}$ cell suspension was diluted into a $2 \mathrm{ml}$ solution for white blood cell (WBC) counts using a NIHOKODEN auto blood cell counter under Pre-dilute $20 \mu \mathrm{l}$ mode. The remaining cells were kept on ice for other experiments.

Flow cytometry and fluorescence-activated cell sorting (FACS). For flow cytometry analysis, 1 million WBCs or Fetal liver cells were stained for $15 \mathrm{~min}$ as indicated in Supplementary Data 9. After staining, cells were washed with PBS once and then resuspended in $500 \mu \mathrm{l}$ PBS for flow cytometry on an LSRII cytometer. Data collection was done with BD FACSDiva software (v8.0.1). The gating strategy for different populations is provided in Supplementary Fig 9

For FACS sorting, mature blood cells were stained with biotin-labeled antilineage surface marker antibodies as indicated in Supplementary Data 9 for $30 \mathrm{~min}$ 
incubation at $4{ }^{\circ} \mathrm{C}$, followed by lineage depletion on an autoMACS machine under "depletes" mode.

Lineage depleted cells were stained as previously described for $15 \mathrm{~min}$. Then cells were washed once and were resuspended to a final cell concentration of $40 \mathrm{M}$ / $\mathrm{ml}$ for FACS on an Arial I/II. Post-sort was performed for double checking of cell populations and for cell number counting. The gating strategy for different populations is provided in Supplementary Fig. 9.

FlowJo was used for data plot of flow cytometry and FACS.

Transplantation. Eight hundred thousand fetal liver cells (isolated from E14.5-day old Znf143 f/+ x Vav1-iCre- or Znf143 f/f x Vav1-iCre+ embryos) or RBC lysis buffer treated BMCs (from Znf143 f/f x Mx1-Cre- or Znf143 f/f x Mx1-Cre+ male mice) were mixed with two hundred thousand RBC lysis buffer treated BMCs isolated from CD45.1 congenic mice to a final cell concentration of ten million per ml. Recipient CD45.1 congenic mice were lethally irradiated (9.5 Gy) using a $\gamma$ irradiator, followed by injecting one million mixed cells per mouse. Every four weeks after injection, $100 \mu \mathrm{l}$ peripheral blood (PB) was collected from each mouse to check for chimerism. To examine chimerism formation, $\mathrm{PB}$ was lysed with $1 \mathrm{ml}$ $\mathrm{RBC}$ lysis buffer for $5 \mathrm{~min}$ at room temperature. After washing with PBS, cells were stained with antibody mixtures as indicated in Supplementary Data 9 for $15 \mathrm{~min}$ at $4^{\circ} \mathrm{C}$, followed by analysis on an LSRII.

RNA isolation, library construction, and next-generation sequencing. The HSC SLAM population (LSK+CD150+CD48-) was sorted into PBS plus FBS and EDTA. After post-sort to confirm the sorted population and to count cell number, cells were spun down for RNA extraction following trizol-chloroform-ethanol extraction with glycogen or glycoblue precipitation. RNA samples with RNA integrity number (RIN) score higher than 8.0 were selected for RNA-seq library construction using SMART Seq v4 Ultra Low Input RNA kit from Takara and Nextera XT DNA library prep kit from Illumina, with PAGE-gel purification for final library size selection. For next-generation sequencing (NGS), 6 libraries (three from Znf143 f/f x Rosa26ERT2-Cre- mice, and three from Znf143 f/f x Rosa26ERT2$\mathrm{Cre}+$ mice, all mice were littermates) were pooled together and were sequenced using a Nextseq high output 150 cycle v2 kit in the Illumina Nextseq 500 platform to obtain at least 50 million $76 \mathrm{bp}$ paired-end reads per RNA library. Illumina bcl2fastq2 Conversion Software (v2.20) was used for Next Generation Sequencing library demultiplexing. Fastqc software was used for data quality check.

Co-immunoprecipitation (co-IP). After post-sort to confirm the sorted population and to count cell numbers, HSPCs (lineage- $c$-kit + ) were washed with PBS once and then lysed in $1 \mathrm{ml}$ IP buffer (50 mM Tris- $\mathrm{Cl} \mathrm{pH} 8.0,100 \mathrm{mM}$ Na Fluoride, 30 mM Na Pyrophosphate, $2 \mathrm{mM}$ Na Molybdate, $5 \mathrm{mM}$ EDTA, $2 \mathrm{mM}$ Na Vanadate, $1 \% \mathrm{NP}-40$, and freshly prepared protease inhibitor purchased from Merk, cat.no. 11697498001 ) on ice for $10 \mathrm{~min}$ with occasionally inverting. After spinning down at $13,200 \mathrm{rpm}$ for $10 \mathrm{~min}$ at $4^{\circ} \mathrm{C}$, the supernatant was transferred into a new tube containing antibody pre-bound dynabeads protein beads for overnight incubation at $4{ }^{\circ} \mathrm{C}$ (anti-CTCF antibody, \#07-729, Millipore; Anti-ZNF143 rabbit serum is generated in our lab); $50 \mu \mathrm{l}$ of the supernatant was stored at $-20^{\circ} \mathrm{C}$ as input. The next day, after washing beads for five times with IP buffer, loading dye was added to the beads and input for boiling at $97^{\circ} \mathrm{C}$ for $10 \mathrm{~min}$, followed by Western-blot analysis.

\section{Chromatin immunoprecipitation (ChIP), library construction, and next-} generation sequencing. ChIP was performed as described previously ${ }^{23}$. Briefly, after post-sort to confirm the sorted population and to count the cell numbers, HSPCs (lineage- c-kit+) were resuspended in PBS to a concentration of $1 \mathrm{M}$ per $\mathrm{ml}$. Cells were fixed with $1 \%$ formaldehyde for $15 \mathrm{~min}$ at RT with rotation. Then formaldehyde was neutralized by adding $2.5 \mathrm{M}$ glycine to a final concentration of $0.125 \mathrm{M}$ and rotating for $5 \mathrm{~min}$ at RT. After washing with PBS, cells were lysed with ChIP SDS lysis buffer (100 mM NaCl,50 mM Tris-Cl pH8.0, 5 mM EDTA, 0.5\% SDS, $0.02 \% \mathrm{NaN}_{3}$, and fresh protease inhibitor), and then stored at $-80{ }^{\circ} \mathrm{C}$ until further processing. Nuclei were collected by spinning down at $13,000 \mathrm{rpm}$ for 10 min. The nuclear pellet was resuspended in IP solution (2 volume ChIP SDS lysis buffer plus 1 volume ChIP triton dilution buffer (100 mM Tris-Cl pH8.6, $100 \mathrm{mM}$ $\mathrm{NaCl}, 5 \mathrm{mM}$ EDTA, $5 \%$ Triton X-100), and fresh proteinase inhibitor) for sonication using either a Bioruptor or Covaris to obtain $200 \mathrm{bp}$ to $500 \mathrm{bp}$ DNA fragments. After spinning down to remove debris, sonicated chromatin was pre-cleared by adding $30 \mu \mathrm{l}$ washed dynabeads protein $\mathrm{A} / \mathrm{G}$ and rotated at $4{ }^{\circ} \mathrm{C}$ for $2 \mathrm{~h}$. Precleared chromatin was incubated with antibody pre-bound dynabeads protein $\mathrm{A} / \mathrm{G}$ overnight at $4{ }^{\circ} \mathrm{C}$ (Anti-ZNF143 rabbit serum is generated in our lab; anti-CTCF antibody, \# 07-729, Millipore; anti-tri-methyl-histone H3 (Lys27) antibody, \#9733BC, Cell Signaling Technology; anti-histone H3 (acetyl K27), \#ab4729, Abcam). Next day, magnetic beads were washed through the following steps: buffer 1 (150 mM NaCl, $50 \mathrm{mM}$ Tris-Cl, $1 \mathrm{mM}$ EDTA, $5 \%$ sucrose, $0.02 \% \mathrm{NaN}_{3}, 1 \%$ Triton X-100, 0.2\% SDS, pH 8.0) two times; buffer 2 (0.1\% deoxycholic acid, $1 \mathrm{mM}$ EDTA, $50 \mathrm{mM}$ HEPES, $500 \mathrm{mM} \mathrm{NaCl}, 1 \%$ Triton X-100, $0.02 \% \mathrm{NaCl}, \mathrm{pH} 8.0$ ) two times; buffer 3 (0.5\% deoxycholic acid, $1 \mathrm{mM}$ EDTA, $250 \mathrm{mM} \mathrm{LiCl}, 0.5 \% \mathrm{NP} 40$, $0.02 \% \mathrm{NaN}_{3}$ ) two times; TE buffer one time. After reverse crosslinking and purification of DNA, qPCR or library construction was performed accordingly.
For library construction, a Thruplex DNA seq kit from Rubicon Genomics was used following the manufacturer's instructions. TBE PAGE-gel size selection was performed for the final library size selection to obtain $250 \mathrm{bp}$ to $500 \mathrm{bp}$ ChIP-sec libraries. Pooled libraries at $10 \mathrm{nM}$ concentration were sequenced in the Illumina Nextseq 500 platform. Illumina bcl2fastq2 Conversion Software (v2.20) was used for Next Generation Sequencing library demultiplexing. The Fastqc software was used for data quality check.

ChIP-reChIP. ZNF143 ChIP was performed as described above until the TE buffer washing step. Then immunocomplexes were eluted by incubating with $50 \mu 110$ $\mathrm{mM}$ DTT for $30 \mathrm{~min}$ at RT. After diluting the elution with $1 \mathrm{ml}$ IP buffer, $20 \mu \mathrm{l}$ CTCF antibody or $5 \mu$ g normal rabbit IgG (\#2729, Cell Signaling Technology) prebound protein A dynabeads were added for overnight rotation at $4{ }^{\circ} \mathrm{C}$, followed by standard ChIP procedure.

In situ HiC library construction. HSPCs (lineage- Sca-1+c-kit+) were used for in situ HiC libraries construction using Arima-HiC kit and Swift biosciences Accel-NGS ${ }^{\oplus} 2 \mathrm{~S}$ plus DNA library kit following the low input procedure instructions.

Chromatin conformation capture (3C). 3C was performed as described previously ${ }^{48}$. In brief, HSPCs (lineage- c-kit+) were crosslinked in $1 \%$ formaldehyde for $15 \mathrm{~min}$ at RT with rotating. Then formaldehyde was neutralized by adding 2.5 $\mathrm{M}$ glycine to a final concentration of $0.125 \mathrm{M}$ and rotating for $5 \mathrm{~min}$ at RT. After washing in PBS, cells were resuspended in $1 \mathrm{ml}$ lysis buffer $(10 \mathrm{mM}$ Tris- $\mathrm{HCl}$ pH8.0, $10 \mathrm{mM} \mathrm{NaCl}, 5 \mathrm{mM}$ EDTA, $0.5 \% \mathrm{NP} 40$, add protease inhibitor freshly) and lysed with a douncer on ice. After spinning down at $2000 \mathrm{rpm}$ for $10 \mathrm{~min}$ at $4{ }^{\circ} \mathrm{C}$, nuclei were washed with $1 \mathrm{X}$ CutSmart buffer from $\mathrm{NEB}$, followed by resuspending in $1.8 \mathrm{ml}$ nuclease free (NF) $\mathrm{H}_{2} \mathrm{O}$. After splitting into 4 tubes containing $60 \mu \mathrm{l}$ of 10X CutSmart buffer and $15 \mu \mathrm{l}$ of $10 \%$ SDS buffer, samples were incubated at $37^{\circ} \mathrm{C}$ $1 \mathrm{~h}$ with shaking, followed by adding $75 \mu \mathrm{l}$ of $20 \%$ Triton X-100 to each tube for 1 -h incubation at $37^{\circ} \mathrm{C}$ with shaking. From each tube $20 \mu \mathrm{l}$ samples were taken out and were combined as "undigested" samples and stored at $-20^{\circ} \mathrm{C}$. HindIII-HF was added into each tube (700 $\mathrm{U}$ per tube) for overnight digestion at $37^{\circ} \mathrm{C}$ with shaking. The next day after confirming a digestion efficiency over $80 \%, 50 \mu \mathrm{g}$ digested DNA were taken out into a new tube and the volume adjusted to $600 \mu \mathrm{l}$ with $\mathrm{NF}_{2} \mathrm{O}$. After adding $80 \mu \mathrm{l} 10 \%$ SDS, samples were incubated at $65^{\circ} \mathrm{C}$ for 25 min. Heat inactivated chromatin was added into ligation mix $(5 \mathrm{ml} 10 \mathrm{X}$ ligation buffer, $2.687 \mathrm{ml} \mathrm{20 \%}$ Triton X-100) and the volume adjusted to $50 \mathrm{ml}$ with $\mathrm{NF} \mathrm{H}_{2} \mathrm{O}$ to a final concentration of $1 \mathrm{ng} / \mu \mathrm{l}$, followed by $1 \mathrm{~h}$ incubation at $37^{\circ} \mathrm{C}$ with rotation at 30 RPM. After adding $990 \mathrm{U}$ T4 DNA ligase (Thermo Scientific), samples were incubated at $16^{\circ} \mathrm{C}$ overnight with rotation at $15 \mathrm{RPM}$. The next day, $132 \mu \mathrm{l}$ of proteinase $\mathrm{K}$ (Ambion) were added into each sample, followed by $65^{\circ} \mathrm{C}$ incubation overnight. The next day, after adding $132 \mu \mathrm{l}$ of RNase A (PureLink), samples were incubated at $37^{\circ} \mathrm{C}$ for an hour, followed by phenol: chloroform DNA purification. Chromatin-chromatin interactions between the Cebpa promoter and $-37 \mathrm{~kb}$ enhancer region and between the Cebpa promoter and $-18 \mathrm{~kb}$ upstream region were examined by $\mathrm{qPCR}$. The $-76 \mathrm{~kb}$ upstream region having no interaction with Cebpa promoter was selected as negative control.

\section{Data analysis}

Dot plots, survival curves. All dot plots and survival curves are drawn with GraphPad Prism unless further indicated in the data analysis section.

RNA-seq data analysis. After trimming of adaptor sequences with TrimGalore, paired-end reads were aligned to mouse genome GRCm 38 (mm10) using STAR ${ }^{49}$ After calculating raw gene expression (raw reads count) by FeatureCounts ${ }^{50}$, normalized profiling results were obtained with DESeq 2 for differential expression analysis $^{51}$. Gene Set Enrichment Analysis (GSEA) was performed using the GSEA pipeline from the Broad Institute ${ }^{52,53}$. For visualization on the UCSC genome browser or IGV, bam files obtained from STAR were first converted into bedgraph format using BEDTools ${ }^{54}$, then converted into bigwig files with UCSC bedGraphtoBigWig program.

ChIP-seq data analysis. After trimming of adaptor sequences by TrimGalore, paired-end reads were aligned to mouse genome GRCm38 (mm10) using bowtie 2 with output in sam file format ${ }^{55}$. SAMtools was used (1) to convert sam file to bam file, (2) to remove both low mapping quality score reads (mapq score less than 20) and PCR duplicates ${ }^{56}$. ChIP-seq peaks were called by MACS 2 with a false discovery

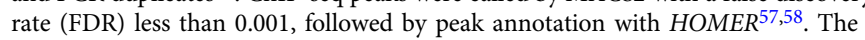
resulting alignments were extended to $150 \mathrm{bp}$ and then converted into signals by BEDTools in bedGraph format ${ }^{54}$. We adjusted the average coverage of each ChIPseq experiment to 1 . Finally, the resulting files were converted to bigwig format by bedGraphToBigWig for visualization and generating heatmaps ${ }^{59}$. Correlation of replicates was assessed with the Deeptools multiBigwigSummary BED-file function 60 . 
Motif discovery. To discover CTCF and ZNF143 motifs in the mouse genome, bed files generated by MACS2 were used for de novo motif scanning using HOMER findMotifsGenome function with default settings ${ }^{58}$. Then the discovered CTCF and ZNF143 motifs were used for whole genome scanning using HOMER scanMotifGenomeWide function with output of bed files. Distances between CTCF and ZNF143 motifs in genome-wide were determined by BEDTools. $R$ package ggplot was applied for result visualization in Rstudio.

ZNF143 mutant motifs were generated by loading ZNF143 motif obtained from HOMER to the online program Regulatory Sequence Analysis Tools (RSAT) with permute-matrix function ${ }^{24}$. Then distances between CTCF and mutant ZNF143 motifs were discovered by BEDTools and were visualized by $R$ in Rstudio.

Motifs orientation. As ZNF143 and CTCF motifs are not palindromic and could locate on either the forward or reverse strand of DNA, a pair consisting of ZNF143 and the nearest CTCF motif have four potential orientations as illustrated in Figure S2C, lower panel: (a) convergent on opposite strands, (b) divergent on opposite strands, (c) both on forward strand (F-F), (d) both on reverse strand (RR). To determine orientations in 10,544 pairs of ZNF143 and CTCF motifs located $37 \mathrm{bp}$ apart, strand specificity of each motif was detected using HOMER, and then each pair of motifs were sub-grouped as described above to get the final count by $R$ in Rstudio.

Promoter and enhancer calling. Active promoters were identified by H3K27ac peaks within $1000 \mathrm{bp}$ to a nearest transcription start site (TSS). Active enhancers were identified by $\mathrm{H} 3 \mathrm{~K} 27 \mathrm{ac}$ peaks that located outside of promoter regions.

\section{Quantification of ChIP-seq signal on promoter, enhancer, or loops anchor} regions. For calculating the average of ChIP-seq signals on a promoter, enhancer, or nearby CTCF motifs within loops anchor regions, HOMER was used to make tag directories of individual ChIP-seq bam files, followed by calculating ChIP-seq signal coverage using HOMER annotatePeaks function ${ }^{58}$. The output was visualized as a mean plot using the $R$ package in Rstudio.

Heatmap of ChIP-seq signal on promoter, enhancer, or loops anchor regions. Deeptools or ngsplot was used to generate heatmap of ChIP-seq on different regions $s^{60,61}$. First, desired bed files indicating such regions were generated as described in this manuscript. Then bigwig files from ChIP-seq data were used to generate a matrix on input regions by Deeptools computeMatrix function, followed by Deeptools plotHeatmap function to draw heatmap. Deeptools was also used for calculating genome-wide correlations among ChIP-seq signals in bigWig files with bin size of $5000 \mathrm{bp}$.

For ngsplot, bam files from ChIP-seq data and desired bed files were used for heatmap generation with ngs.plot.r function.

HiC data analysis. HiC contact matrices were generated using the Juicer tools suite (v1.9.9_jcuda.0.8) following the instruction ${ }^{7}$. The Knight-Ruiz balanced observed/ expected interaction frequencies were calculated at $2.5 \mathrm{Mb}, 1 \mathrm{Mb}, 500 \mathrm{~kb}, 250 \mathrm{~kb}$, $100 \mathrm{~kb}, 50 \mathrm{~kb}, 25 \mathrm{~kb}$, and $5 \mathrm{~kb}$ resolution. The matrices were visualized in Juicebox (v1.9.8). The eigenvector values were calculated at $1 \mathrm{Mb}$ resolution using Juicer tools eigenvector function. The PC1 values were calculated using HOMER following its $\mathrm{HiC}$ analysis tutorial ${ }^{62}$.

Insulation scores were calculated as described ${ }^{28}$. Dense contact matrices for each chromosome were extracted from.hic files using Juicer tools dump function returning the observed contacts without normalization at $25 \mathrm{~kb}$ resolution. The resulting matrices were transformed into the $\mathrm{HiC}-\mathrm{PRO}$ format ${ }^{63}$ using a custom $R$ script. The insulation score was calculated from these $H i C-P R O$ contact matrices using the the matrix2insulation.pl function in $\mathrm{HiC}-\mathrm{PRO} \mathrm{O}^{28}$. The resulting insulation score per chromosome was aggregated into a single bedGraph and then transformed to bigwig using the bedGraphToBigWig function from UCSC together with the chromosome size information for $\mathrm{mm} 10$ in UCSC. Profiles of genomewide insulation score around TADs boundaries were visualized by Deeptools.

TADs were called using the Juicer tools Arrowhead algorithm at $25 \mathrm{~kb}$ resolution. Loops were called using Juicer tools Hiccups function at $5 \mathrm{~kb}$ resolution. Loops intensities were calculated by Juicer tools dump function with input of.hic files generated by Juicer tools. Aggregate peak analysis on subgroup loops was performed using Juicer tools APA function, and the result is visualized with $R$ package in Rstudio. To discover CTCF motifs on loop anchors, bedpe files containing loops information were examined by Juicer tools MotifFinder function.

Reporting summary. Further information on research design is available in the Nature Research Reporting Summary linked to this article.

\section{Data availability}

The ChIP-seq, RNA-seq, and HiC data that support the findings of this study have been deposited in GEO with the GEO accession number "GSE144712". Biological material used in this study can be obtained from the authors upon request. The authors declare that all other data supporting the findings of this study are available within the paper and its supplementary information files. Source data are provided with this paper.
Received: 30 April 2020; Accepted: 23 November 2020; Published online: 04 January 2021

\section{References}

1. $\mathrm{Yu}, \mathrm{M}$. \& Ren, B. The three-dimensional organization of mammalian genomes. Annu. Rev. Cell Dev. Biol. 33, 265-289 (2017).

2. Dekker, J., Marti-Renom, M. A. \& Mirny, L. A. Exploring the threedimensional organization of genomes: interpreting chromatin interaction data. Nat. Rev. Genet. 14, 390-403 (2013).

3. Lee, T. I. \& Young, R. A. Transcriptional regulation and its misregulation in disease. Cell 152, 1237-1251 (2013).

4. Dixon, J. R. et al. Topological domains in mammalian genomes identified by analysis of chromatin interactions. Nature 485, 376-380 (2012).

5. Nora, E. P. et al. Spatial partitioning of the regulatory landscape of the Xinactivation centre. Nature 485, 381-385 (2012).

6. Zuin, J. et al. Cohesin and CTCF differentially affect chromatin architecture and gene expression in human cells. Proc. Natl Acad. Sci. USA 111, 996-1001 (2014)

7. Rao, S. S. et al. A 3D map of the human genome at kilobase resolution reveals principles of chromatin looping. Cell 159, 1665-1680 (2014).

8. Tang, Z. et al. CTCF-mediated human 3D genome architecture reveals chromatin topology for transcription. Cell 163, 1611-1627 (2015).

9. Nora, E. P. et al. Targeted degradation of CTCF decouples local insulation of chromosome domains from genomic compartmentalization. Cell 169, 930-944 e922 (2017).

10. Ren, G. et al. CTCF-mediated enhancer-promoter interaction is a critical regulator of cell-to-cell variation of gene expression. Mol. Cell 67, 1049-1058 e1046 (2017).

11. Weintraub, A. S. et al. YY1 is a structural regulator of enhancer-promoter loops. Cell 171, 1573-1588 e1528 (2017).

12. Kim, T. H. et al. Analysis of the vertebrate insulator protein CTCF-binding sites in the human genome. Cell 128, 1231-1245 (2007).

13. Phillips, J. E. \& Corces, V. G. CTCF: master weaver of the genome. Cell 137, 1194-1211 (2009)

14. Ji, X. et al. 3D chromosome regulatory landscape of human pluripotent cells. Cell Stem Cell 18, 262-275 (2016).

15. Schwarzer, W. et al. Two independent modes of chromatin organization revealed by cohesin removal. Nature 551, 51-56 (2017).

16. Li, Y. et al. The structural basis for cohesin-CTCF-anchored loops. Nature https://doi.org/10.1038/s41586-019-1910-z (2020).

17. Myslinski, E., Krol, A. \& Carbon, P. ZNF76 and ZNF143 are two human homologs of the transcriptional activator Staf. J. Biol. Chem. 273, 21998-22006 (1998)

18. Schuster, C., Myslinski, E., Krol, A. \& Carbon, P. Staf, a novel zinc finger protein that activates the RNA polymerase III promoter of the selenocysteine tRNA gene. EMBO J. 14, 3777-3787 (1995).

19. Wang, $\mathrm{H}$. et al. Genome-wide analysis reveals conserved and divergent features of Notch1/RBPJ binding in human and murine T-lymphoblastic leukemia cells. Proc. Natl Acad. Sci. USA 108, 14908-14913 (2011).

20. Ngondo-Mbongo, R. P., Myslinski, E., Aster, J. C. \& Carbon, P. Modulation of gene expression via overlapping binding sites exerted by ZNF143, Notch1 and THAP11. Nucleic Acids Res 41, 4000-4014 (2013).

21. Heidari, N. et al. Genome-wide map of regulatory interactions in the human genome. Genome Res. 24, 1905-1917 (2014).

22. Bailey, S. D. et al. ZNF143 provides sequence specificity to secure chromatin interactions at gene promoters. Nat. Commun. 2, 6186 (2015).

23. Gonzalez, D. et al. ZNF143 protein is an important regulator of the myeloid transcription factor C/EBPalpha. J. Biol. Chem. 292, 18924-18936 (2017).

24. Nguyen, N. T. T. et al. RSAT 2018: regulatory sequence analysis tools 20th anniversary. Nucleic Acids Res. 46, W209-W214 (2018).

25. Kim, S. et al. Probing allostery through DNA. Science 339, 816-819 (2013).

26. Guo, Y. et al. CRISPR inversion of CTCF sites alters genome topology and enhancer/promoter function. Cell 162, 900-910 (2015).

27. de Wit, E. et al. CTCF binding polarity determines chromatin looping. Mol. Cell 60, 676-684 (2015).

28. Crane, E. et al. Condensin-driven remodelling of $\mathrm{X}$ chromosome topology during dosage compensation. Nature 523, 240-244 (2015).

29. Ogilvy, S. et al. Promoter elements of vav drive transgene expression in vivo throughout the hematopoietic compartment. Blood 94, 1855-1863 (1999).

30. Orkin, S. H. Diversification of haematopoietic stem cells to specific lineages. Nat. Rev. Genet. 1, 57-64 (2000).

31. Bluteau, O. et al. A landscape of germ line mutations in a cohort of inherited bone marrow failure patients. Blood 131, 717-732 (2018).

32. Fedoriw, A. M., Stein, P., Svoboda, P., Schultz, R. M. \& Bartolomei, M. S. Transgenic RNAi reveals essential function for CTCF in $\mathrm{H} 19$ gene imprinting. Science 303, 238-240 (2004) 
33. Heath, H. et al. CTCF regulates cell cycle progression of alphabeta $\mathrm{T}$ cells in the thymus. EMBO J. 27, 2839-2850 (2008).

34. Xie, X. et al. Systematic discovery of regulatory motifs in conserved regions of the human genome, including thousands of CTCF insulator sites. Proc. Natl Acad. Sci. USA 104, 7145-7150 (2007).

35. Cuddapah, S. et al. Global analysis of the insulator binding protein CTCF in chromatin barrier regions reveals demarcation of active and repressive domains. Genome Res. 19, 24-32 (2009).

36. Saldana-Meyer, R. et al. RNA interactions are essential for CTCF-mediated genome organization. Mol. Cell 76, 412-422 e415 (2019).

37. Hansen, A. S. et al. Distinct classes of chromatin loops revealed by deletion of an RNA-binding region in CTCF. Mol. Cell 76, 395-411 e313 (2019).

38. Barisic, D., Stadler, M. B., Iurlaro, M. \& Schubeler, D. Mammalian ISWI and SWI/SNF selectively mediate binding of distinct transcription factors. Nature 569, 136-140 (2019).

39. Krietenstein, N. et al. Ultrastructural details of mammalian chromosome architecture. Mol. Cell 78, 554-565 e557 (2020).

40. Hsieh, T. S. et al. Resolving the 3D landscape of transcription-linked mammalian chromatin folding. Mol. Cell 78, 539-553 e538 (2020).

41. Viny, A. D. et al. Dose-dependent role of the cohesin complex in normal and malignant hematopoiesis. J. Exp. Med 212, 1819-1832 (2015).

42. Viny, A. D. et al. Cohesin members Stag1 and Stag2 display distinct roles in chromatin accessibility and topological control of HSC self-renewal and differentiation. Cell Stem Cell 25, 682-696 e688 (2019).

43. Numata, A. et al. Lysine acetyltransferase Tip60 is required for hematopoietic stem cell maintenance. Blood https://doi.org/10.1182/blood.2019001279 (2020).

44. Sun, S. et al. Chromatin remodeler Znhit1 preserves hematopoietic stem cell quiescence by determining the accessibility of distal enhancers. Leukemia https://doi.org/10.1038/s41375-020-0988-5 (2020).

45. Aranda-Orgilles, B. et al. MED12 regulates HSC-specific enhancers independently of mediator kinase activity to control hematopoiesis. Cell Stem Cell 19, 784-799 (2016).

46. Kuhn, R., Schwenk, F., Aguet, M. \& Rajewsky, K. Inducible gene targeting in mice. Science 269, 1427-1429 (1995).

47. Ventura, A. et al. Restoration of p53 function leads to tumour regression in vivo. Nature 445, 661-665 (2007)

48. Dekker, J., Rippe, K., Dekker, M. \& Kleckner, N. Capturing chromosome conformation. Science 295, 1306-1311 (2002)

49. Dobin, A. et al. STAR: ultrafast universal RNA-seq aligner. Bioinformatics 29, 15-21 (2013)

50. Liao, Y., Smyth, G. K. \& Shi, W. featureCounts: an efficient general purpose program for assigning sequence reads to genomic features. Bioinformatics $\mathbf{3 0}$, 923-930 (2014).

51. Love, M. I., Huber, W. \& Anders, S. Moderated estimation of fold change and dispersion for RNA-seq data with DESeq2. Genome Biol. 15, 550 (2014).

52. Subramanian, A. et al. Gene set enrichment analysis: a knowledge-based approach for interpreting genome-wide expression profiles. Proc. Natl Acad. Sci. USA 102, 15545-15550 (2005).

53. Mootha, V. K. et al. PGC-1alpha-responsive genes involved in oxidative phosphorylation are coordinately downregulated in human diabetes. Nat. Genet 34, 267-273 (2003).

54. Quinlan, A. R. \& Hall, I. M. BEDTools: a flexible suite of utilities for comparing genomic features. Bioinformatics 26, 841-842 (2010).

55. Langmead, B. \& Salzberg, S. L. Fast gapped-read alignment with Bowtie 2. Nat. Methods 9, 357-359 (2012).

56. $\mathrm{Li}, \mathrm{H}$. et al. The sequence alignment/map format and SAMtools. Bioinformatics 25, 2078-2079 (2009).

57. Zhang, Y. et al. Model-based analysis of ChIP-Seq (MACS). Genome Biol. 9, R137 (2008).

58. Heinz, S. et al. Simple combinations of lineage-determining transcription factors prime cis-regulatory elements required for macrophage and B cell identities. Mol. Cell 38, 576-589 (2010).
59. Tan, H. K. et al. DNMT3B shapes the mCA landscape and regulates mCG for promoter bivalency in human embryonic stem cells. Nucleic Acids Res. 47, 7460-7475 (2019).

60. Ramirez, F. et al. deepTools2: a next generation web server for deepsequencing data analysis. Nucleic Acids Res. 44, W160-W165 (2016).

61. Shen, L., Shao, N., Liu, X. \& Nestler, E. ngs.plot: Quick mining and visualization of next-generation sequencing data by integrating genomic databases. BMC Genomics 15, 284 (2014).

62. Heinz, S. et al. Transcription elongation can affect genome 3D structure. Cell 174, 1522-1536 e1522 (2018).

63. Servant, N. et al. HiC-Pro: an optimized and flexible pipeline for Hi-C data processing. Genome Biol. 16, 259 (2015).

\section{Acknowledgements}

The authors thank Michelle Mok Meng Huang and Chelsia Qiuxia Wang for assistance with flow cytometry experiments. This research was supported by the Singapore Ministry of Health's National Medical Research Council under its Singapore Translational Research (STaR) Investigator Award, and by the National Research Foundation Singapore and the Singapore Ministry of Education under its Research Centres of Excellence initiative, as well as NIH grants 1R35CA197697 and P01 HL131477 to D.G.T., and Xiu Research Fund to L.C.

\section{Author contributions}

D.G.T initiated the project and provided guidance throughout. D.G.T, Q.Z, S.L., and M.Y designed the experiments. Q.Z planned and performed molecular, cellular, and mouse experiments. M.G. and Z.H.T participated in mouse experiments. Q.Z, R.T.M, B.L., and L.K. performed bioinformatics analysis. Q.Z, M.Y, M.J.F, M.O, T.B, B.R, and D.G.T interpreted the data. Q.Z. and D.G.T. wrote the manuscript with input from M.Y., L.C., A.N., and B.R

\section{Competing interests}

B.R. is a co-founder of Arima Genomics, Inc. All other authors declare no competing interests.

\section{Additional information}

Supplementary information is available for this paper at https://doi.org/10.1038/s41467020-20282-1.

Correspondence and requests for materials should be addressed to D.G.T.

Peer review information Nature Communications thanks John Pimanda and the other, anonymous, reviewer(s) for their contribution to the peer review of this work. Peer reviewer reports are available.

Reprints and permission information is available at http://www.nature.com/reprints

Publisher's note Springer Nature remains neutral with regard to jurisdictional claims in published maps and institutional affiliations.

Open Access This article is licensed under a Creative Common Attribution 4.0 International License, which permits use, sharing, adaptation, distribution and reproduction in any medium or format, as long as you give appropriate credit to the original author(s) and the source, provide a link to the Creative Commons license, and indicate if changes were made. The images or other third party material in this article are included in the article's Creative Commons license, unless indicated otherwise in a credit line to the material. If material is not included in the article's Creative Commons license and your intended use is not permitted by statutory regulation or exceeds the permitted use, you will need to obtain permission directly from the copyright holder. To view a copy of this license, visit http://creativecommons.org/ licenses/by/4.0/.

(C) The Author(s) 2021 